\title{
Failure load prediction for fiber-reinforced composites based on acoustic emission
}

MARKUS G. R. SAUSE, STEFAN SCHMITT, SINAN KALAFAT

University of Augsburg, Institute for Materials Resource Management, Mechanical Engineering, D-86135 Augsburg

\begin{abstract}
In the design and quality control of fiber-reinforced structures, testing on coupon level and structure level are frequently carried out. In order to accept or reject a final product or material charge, means of quality control are carried out. In safety relevant structures, this is often based on holding a certain proof load. Acoustic emission is already used for the monitoring during proof load testing, but is only used for simple accept/reject diagnosis. For the accepted components typically no assessment is made for the expected residual capacity. We propose an acoustic emission based approach able to perform prediction of the ultimate strength values and to evaluate the materials present stress exposure while being tested. We base our approach on accepted acoustic emission measures, such as the Felicity ratio or the Shelby ratio to assess the structural integrity. Using a combination of an artificial neural network to predict the materials present stress exposure and a simple linear extrapolation we are able to predict the failure strength within the margin of prediction error for all test cases studied. The approach is benchmarked for three types of specimens, systematically changing test volume and load condition. We used tensile tests on fiber-reinforced thermoplastic tape samples, classical tensile test samples and bearing strength samples, all made from the same material.
\end{abstract}

\section{Introduction}

The design of structures from fiber-reinforced composites is one challenging task as soon as a precise calculus of failure modes or failure strength is involved. A multitude of theoretical concepts exist today in order to aid the engineer to predict the occurrence of failure, yet none of them outperforms the other in a general load situation [1-3]. Practically, the uncertainty caused in the design process raises a huge number of testing efforts ranging from small-scale specimens to large-scale structures in order to achieve a level of confidence in the particular material and the particular design. Apart from the aspect of testing costs involved, the assessment of the ultimate load by way of a quality control loading usually obstructs the assessment, as the structure is no longer usable when loaded close to ultimate load. Accordingly, several attempts were made in the past to predict the ultimate load based on data collected during loading to a lower load level.

One data analysis technique that was frequently used to this end is the acoustic emission analysis. During rapid internal displacements, such as during crack initiation and growth, elastic stress-waves are 
released. These propagate in the ultrasonic range and can be detected by sensitive piezoelectric sensing systems. For the specific implications of measurements on fiber-reinforced materials a comprehensive introduction is found in $[4,5]$.

Several concepts have been used to perform acoustic emission based assessment of failure loads, many of them resulting from the need to predict burst pressure levels of fiber-reinforced vessels. The usual load schedule applied (according to e.g. ASTM E 1067) is a cyclic load / unload scheme as seen in figure 1. The earliest application of this schedule in combination with acoustic emission analysis goes back to J. Kaiser in 1950 [6]. He demonstrated for the case of metallic materials, that the acoustic emission usually re-initiates after exceeding the previously reached load level. This behavior of materials has been termed Kaiser-effect to honor his pioneering work in the field of acoustic emission. However, for fiber-reinforced materials the acoustic emission signals often initiate at lower load levels than previously achieved. This fundamental observation goes back to the work of T. Fowler $[7,8]$. Since the first publication in 1977 , the evaluation of the Felicity ratio became an important concept to understand the progression of failure in fiber-reinforced materials using acoustic emission.

As similar measure for damage progression in composite materials, the Shelby ratio has been proposed by K. Downs [9]. It is different from the Felicity ratio in that the AE evaluation is based on the hits detected during un-loading of the test item. When reaching a certain evaluation criterion (such as a total number of accumulated hits) during the un-loading phase the corresponding load level is used for the evaluation. This relates to the previously reached maximum load. This approach has been used i.e. to assess the quality of composite pressure vessels since those with prior damage generate noticeable amounts of $A E$ during de-pressurization [9].

Both quantities, Felicity ratio and Shelby ratio were correlated with the burst pressure of fiber-reinforced composite vessels and indicated that their values are highly correlated [7-11]. Based on this observation J. Waller et al. extended this approach into a forward prediction routine, collecting the Felicity ratio for a certain number of cycles to failure [12]. They proposed to establish a critical Felicity ratio as material property and evaluated their concept for fiber-reinforced strands and pressure vessels.

An another approach to predict the ultimate load of fiber-reinforced composite vessels was proposed by E. Hill and J. Walker using the recorded acoustic emission amplitude distributions as input data [13-15]. They used artificial neural networks to classify the acoustic emission data and based their forward prediction on a second supervised training stage on the entirety of acoustic emission data. This provided 
evidence, that artificial neural networks are flexible enough to perform such predictive tasks. A similar approach has also been applied by other groups for prediction of tensile coupon strength [16].

\section{Failure prediction concept}

Based on these earlier attempts on prediction of composite failure, we propose a modified version of these two principle approaches, effectively combining the strength of the previous attempts. The first part is the data reduction during cyclic loading to meaningful acoustic emission based ratios (e.g. Felicity ratios). Second is the use of these ratios within an artificial neural network to perform the prediction of the expected load status of the structure. Final part is the extrapolation of the load ratios as predictive approach.

Since the present approach uses a supervised classification scheme for artificial neuronal networks, the whole concept subdivides in two stages. The first stage is the generation of a test database to establish the relationship between acoustic emission parameters and load levels. The second stage is the application of the established relationship to a new test item to perform a forward prediction.

For the first stage, the steps are:

1. Definition of acoustic emission parameters to evaluate in each cycle

2. Evaluation of these parameters during load/unload cycles until failure

3. Establishing a relationship between acoustic emission parameters and ratio of load-to-failure

For the second stage, the schedule is slightly different:

1. Definition of acoustic emission parameters to evaluate in each cycle

2. Evaluation of these parameters during load/unload cycles until certain load level

3. Using established relationship to predict ratio of load-to-failure in each cycle

4. Extrapolating the load-to-failure ratio to unity thereby predicting the failure level and adding an estimation of prediction error

As for the importance of the implementation of this schedule, we briefly explain the proposed load/unload schedule. Next, we briefly present the acoustic emission criteria used in this study and elaborate the background why these were chosen instead of others. In the subsequent section, we discuss the approach to implement the relationship between acoustic emission parameters and ratio of 
load-to-failure using artificial neural networks. Finally, the details for the proposed extrapolation and error estimation rules are presented.

\subsection{Cyclic load schedule}

Apart from monotonically increasing the applied load, repetitive loading and unloading cycles according to ASTM E 1067 are a popular concept used in acoustic emission testing. The two principle types of this testing scheme are shown in figure 1 . In both cases, the specimen is subject to a monotonic increasing load until a certain load level $L_{1}$ is reached. Then the specimen is fully unloaded (figure 1-a) or partially unloaded (figure 1-b). In the next "cycle" the load is increased again until a certain load level $L_{2}$ is reached, which exceeds the previously reached load level. This process is repeated N-times up to a certain maximum load level $L_{\max }$ or up to specimen failure $L_{\text {failure }}$. This gives rise to the definition of the load ratio $L R$ for cycle $N$ :

$L R=L_{N} / L_{\text {failure }}$

In the sense of failure criteria of fiber-reinforced materials, this load ratio is the global stress exposure of the structure (cf. Puck's failure theory $[17,18])$, which is also known as reserve factor, when taking the inverse.

In order to conform with the definition of the acoustic emission parameters in the next section the minimum unload level $L_{N, \text { low }}$ needs to be small enough to allow the measurement of small enough ratios, e.g. to measure a Felicity ratio of 0.7 , the requirement is $L_{N, \text { low }}<0.7 \cdot L_{N-1}$.

(a)

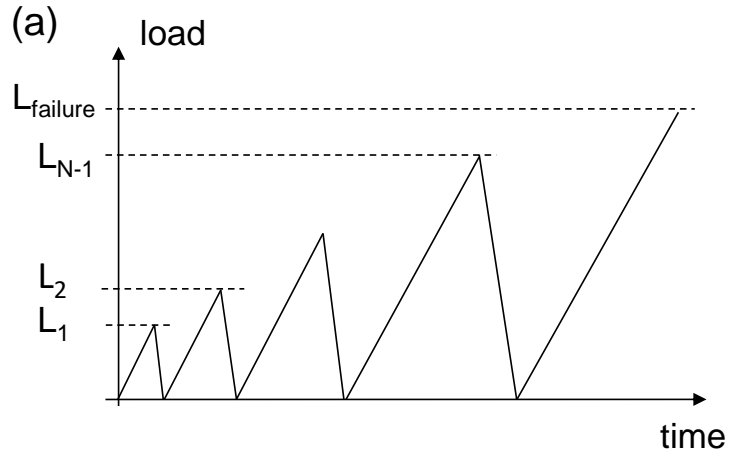

(b)

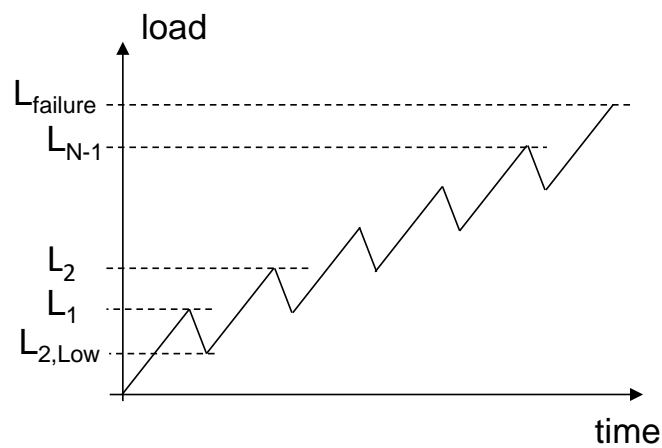

Figure 1: Scheme for definition of load-unload cycles with full unload (a) and partial unload (b).

\subsection{Acoustic emission parameters}

During the loading of the material, acoustic emission signals are recorded simultaneously. For the later application in forward prediction it is important to define parameters such, that they can be calculated in the present load cycle based on input of only the past cycles. Thus, any normalization of values by the number of hits, amplitudes or energies of future cycles is not meaningful for a forward prediction attempt. Furthermore, we propose to use only parameters, which have intrinsic normalization. That is, 
they are ratios instead of absolute values. This should make the numerical values of these parameters less susceptible to changes of test volume and possibly test condition (see section 4).

\subsubsection{Felicity ratio}

One such parameter that falls into this category is the well-established Felicity ratio. As seen in figure 2a below, the Felicity ratio is defined for every cycle $N>1$ using the onset of acoustic emission hits in the present cycle $L_{F R, N}$ and relates this value to the previously reached load $L_{N-1}$ :

$F R_{N}=L_{F R, N} / L_{N-1}$

One particular challenge arises from the correct measurement of the onset of acoustic emission. In standards like ASTM E 1067 the term "significant" is used without further clarification. In general, the best measure of an acoustic emission onset may not necessarily be identical to the occurrence of the first signal, since this may likely be a noise signal or a premature (unique) local failure on the microscale, which is not representative for the full specimen or full structure under load. To decrease the susceptibility to such outliers, one approach is the use of the mean onset value of the first $K$ hits $[9,10]$. To avoid setting $K$ as absolute value, we propose to use an adaptive $K$ value, based on the number of hits detected in the present cycle, i.e. at $5 \%$ of the hits detected in the present cycle.

For cases, where there are many acoustic emission hits in each cycle, curve-fitting approaches can be considered. As specifically proposed by [19], trend monitoring using the exponentially weighted moving average of the activity curves may yield an improved approach to detect the onset of acoustic emission. Especially for cases, where the first hits show negligible energy compared to the rest of the recorded hits this may yield more stable and meaningful onset values. Another curve fitting approach is the backwards extrapolation of the accumulated number of acoustic emission hits, if this curve is relatively linear as function of load. In $[11,20]$ the Historic index was also proposed as a measure of an acoustic emission onset (definition see e.g. DIN EN 15857). As schematically outlined in figure 2-b, each approach may lead to a different onset value. However, the sequence of onsets shown in the graph is not representative of the particular approach, e.g. Historic index does not need to be the last of the onsets.

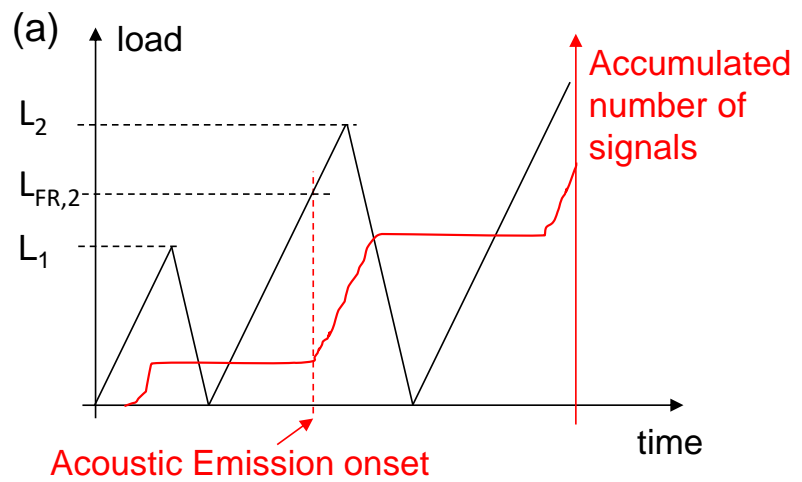

(a)

Acoustic Emission onset

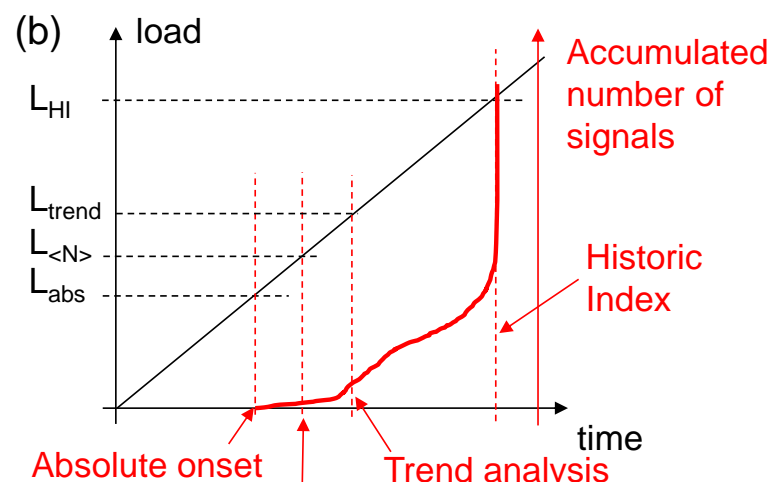

Mean value of first $\mathrm{N}$ signals 
Figure 2: Scheme for definition of Felicity ratio (a) and established ways to measure the acoustic emission onset (b).

Within this study, we define the Felicity ratio as relative shares for fractions $5 \%, 10 \%, 15 \%$ and $20 \%$ of the total number of hits during the ascending part of the cycle. This is denominated FR5, FR10, FR15 and FR20 in the following. In addition, we define the average Felicity ratio as arithmetic mean of these for values:

$$
\langle F R\rangle=\frac{1}{4}(F R 5+F R 10+F R 15+F R 20)
$$

Therefore, in total five different Felicity ratios are evaluated for each load cycle.

\subsubsection{Shelby ratio}

Another criterion that is applicable for the proposed scenario is the Shelby ratio. Other than the Felicity ratio, the Shelby ratio is evaluated during the unloading phase of the present load cycle. In the case of detection of unloading acoustic emission, the Shelby ratio is defined as:

$S R_{N}=\Delta L_{S R, N} / L_{N}$

The definition uses the unloading range $\Delta L_{S R, N}$ at the instant of reaching a critical number of acoustic emission hits $N_{\text {crit }}$ (cf. figure 3). This is than related to the previously reached peak load, i.e. the maximum of the present cycle $L_{N}$. In analogy to the definition of the Felicity ratio, the unloading range can be established based on a fixed number of hits, by relative shares, e.g. at reaching $95 \%$ of the number of unloading hits or by trend analysis methods as described above.

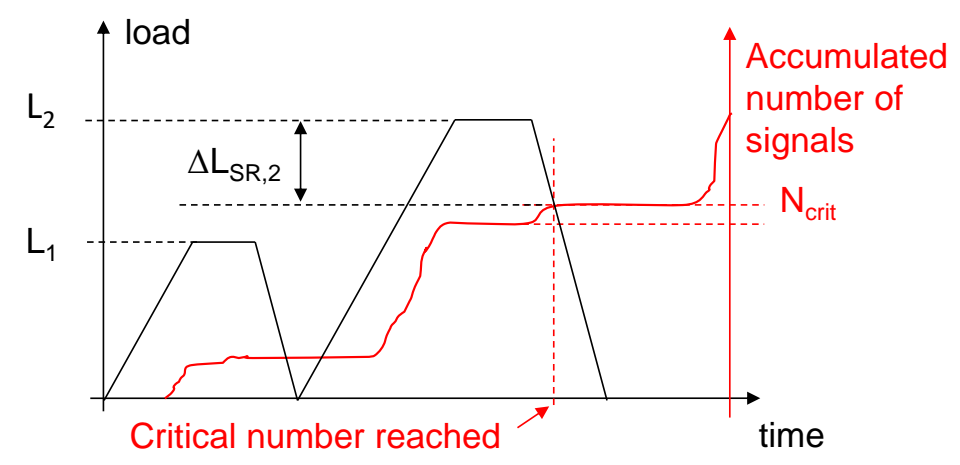

Figure 3: Scheme for definition of Shelby ratio.

Herein, we follow the same concept for the Shelby ratio as for the Felicity ratio. We define the number of critical hits as relative shares for fractions of $95 \%, 90 \%, 85 \%$ and $80 \%$ of the total number of hits during the descending part of the cycle. This is denominated SR95, SR90, SR85 and SR80 in the following. In addition, we define the average Shelby ratio as arithmetic mean of these for values:

$\langle S R\rangle=\frac{1}{4}(S R 95+S R 90+S R 85+S R 80)$ 
Therefore, in total five different Shelby ratios are evaluated for each load cycle.

\subsubsection{Energetic ratios}

In addition to the two previous ratios, which are based on the number of hits it appears feasible to define a measure for the acoustic emission onset by means of energetic signal quantities, as it is generally assumed, that the intensity of the signal is related to the severity of the damage formation.

The primary quantities that are used to describe the energetic relevance of an acoustic emission signal are the maximal signal amplitude, the signal energy as integrated squared signal amplitude divided by input impedance of measurement system and the "Root-Mean-Square" (RMS) value as square-root of the mean squared signal amplitude over the duration of the hit. As schematic example for these features the accumulated curve of one of these values is shown in figure 4. Following the thought of selfreferencing values, the onset is defined as load value $L_{E, N}$ where the previously reached accumulated value $A_{N-1}$ is exceeded, i.e. at the value $A_{N} \geq 2 \cdot A_{N-1}$.

$E R_{N}=L_{E, N} / L_{N-1}$

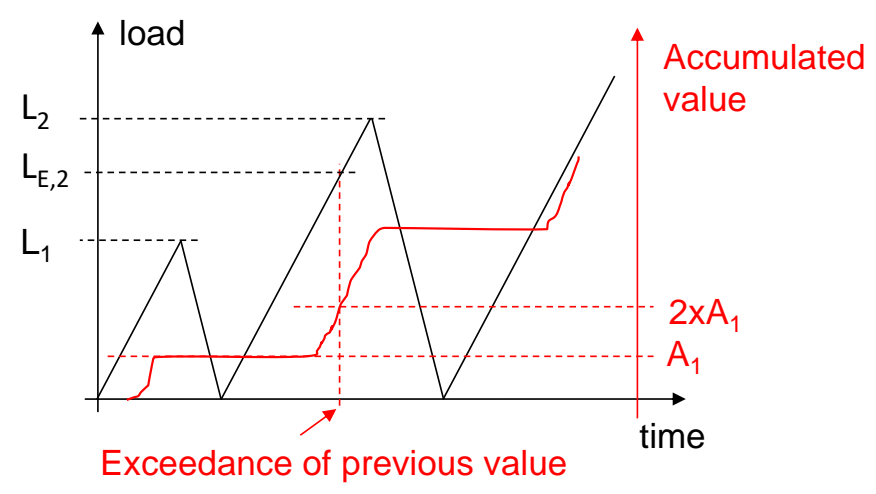

Figure 4: Scheme for definition of the energetic ratios.

In this study, we use the acoustic emission parameters signal amplitude in $\left[\mathrm{dB}_{\mathrm{AE}}\right]$, the absolute signal energy in [aJ] and the average signal level in $\left[\mathrm{dB}_{\mathrm{AE}}\right]$ to establish the accumulated values. Accordingly, we evaluate one such ratio for each quantity, i.e. ERAMP, ERENE and ERASL.

\subsection{Linking acoustic emission and failure load}

The next step is the generation of a symbolic relationship between a particular combination of acoustic emission parameters and the measured load ratios. Using one input parameter like the Felicity ratio and one output parameter like the load ratio, this can be done by an analytical relationship between these two values. In the work of $[12,19]$ this was achieved using a linear regression analysis. The disadvantage of this approach consists in the necessity of a strict analytical relationship and the strong impact of data scatter, which will affect the forward prediction capabilities (see section 2.4). Consequently, it appears useful to define a function with multiple input parameters to fit the single output parameter (load ratio): 
This type of function approximation problem can be addressed by multiple techniques of multivariate data analysis (see e.g. [21]). In this work, we use a multi-layer feedforward neural network implemented in MATLAB to perform the function approximation using a Levenberg-Marquardt algorithm. We use the 13 different acoustic emission ratios as input values of an artificial neural network applying a linear transfer function for each neuron to fit the corresponding load ratio. For the present purpose, a network structure with 5 to 10 neurons per layer and one or two hidden layers proved sufficient to reach a reasonable function approximation. The specific settings depend on the complexity faced (see section 4). To avoid overfitting the data, $70 \%$ of the input data values are used as training set, whereas another $15 \%$ are used for the validation of the algorithm convergence and the remaining $15 \%$ for evaluation of the performance of the artificial neural network. For the latter, the mean-square error of the fit between input values and output values is applied. The full procedure is repeated 100 times for one configuration with new random selection of training, validation and test datasets and the artificial neural network with best performance is selected based on the lowest mean-square error.

After the training step, the artificial neural network $f_{\text {opt }}$ can be used to predict the expected load ratio for a certain combination of acoustic emission input parameters.

\subsection{Forward prediction routine}

After an established relationship between acoustic emission parameters and load ratio is available, it is feasible to apply the procedure for forward prediction. At a given load cycle $N$ we can calculate the acoustic emission parameters, and apply:

$$
L R_{N}=f_{o p t}\left(F R_{N}, S R_{N}, E R_{N}, \ldots\right)
$$

This allows to predict the load ratio of cycle $N$. Knowing the actual peak load $L_{N}$ this allows to relate the present peak load $L_{N}$ and the predicted load ratio $L R_{N}$ as seen in figure 5. For a proper function $f_{\text {opt }}$ we expect a linear relationship between the $L R$ and $L_{N}$, so it is appropriate to perform a linear regression for the data points, which follows the idea of earlier work reported by Waller et al. [12]. For our implementation we consider the linear regression slope $m$ with forced intercept at the origin and calculate the linear regression error $\varepsilon$ as

$\varepsilon=\sqrt{\frac{\left(\sum L R_{N}-m \cdot L_{N}\right)^{2}}{N-2}} \cdot\left(\frac{N}{\left(N \cdot \sum L_{N}^{2}\right)-\left(\sum L_{N}\right)^{2}}\right)$

For the purpose of forward prediction, the extrapolation of the linear regression to $L R=1$ yields the corresponding predicted failure load. Based on the scatter of the data points there is an uncertainty in the forward prediction, which appears appropriate to be considered in the load prediction values. With 
the constraints of $L=0$ implies $L R=0$, the extrapolation function has to pass the origin. Accordingly, we do not apply a confidence interval approach, but use $\varepsilon$ as upper and lower bound for the slope $m$. The intersections of $(m \pm \varepsilon) \cdot L$ with $L R=1$ are taken as uncertainty of the failure prediction value.

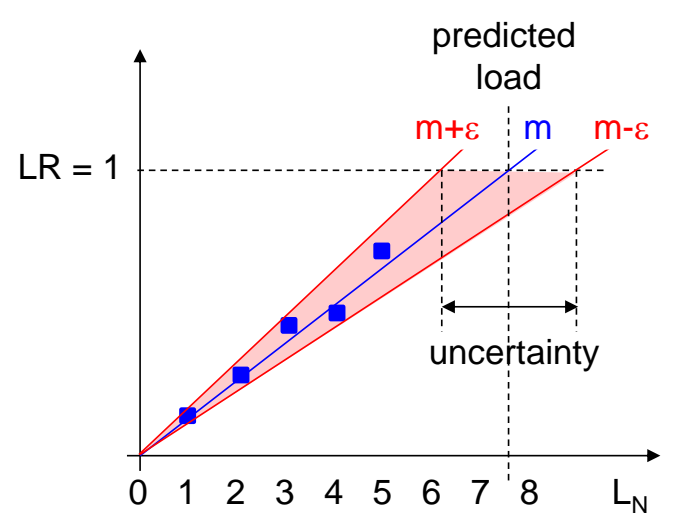

Figure 5: Scheme for extrapolation of $L_{N}$ values to predict failure load.

\section{Experimental}

For the experimental work, we used fiber-reinforced thermoplastic composites made from Torayca T700S $12 \mathrm{k}$ carbon fibers and PPS matrix material. For the evaluation of the approach we use three different types of mechanical tests, which are feasible to carry out in load/unload cycles. To demonstrate the flexibility of the approach to adapt to different failure modes, we use data from tensile tests and load bearing tests. To incorporate volume effects, which could be problematic for the evaluation of acoustic emission parameters, we use tensile tests on tape material, classical tensile test specimens and load bearing test geometries of two sizes as seen in figure 6. The corresponding test volumes and statistics are reported in table 1, including the average accumulated acoustic emission energy of each test condition. The number of acoustic emission sensors was chosen based on attenuation measurements of the samples to ensure equal sensitivity in each configuration. In the following, we briefly describe the test condition of each configuration. All specimens were tested in standard climate condition $23{ }^{\circ} \mathrm{C}$ temperature and $50 \%$ relative humidity. 
(a)

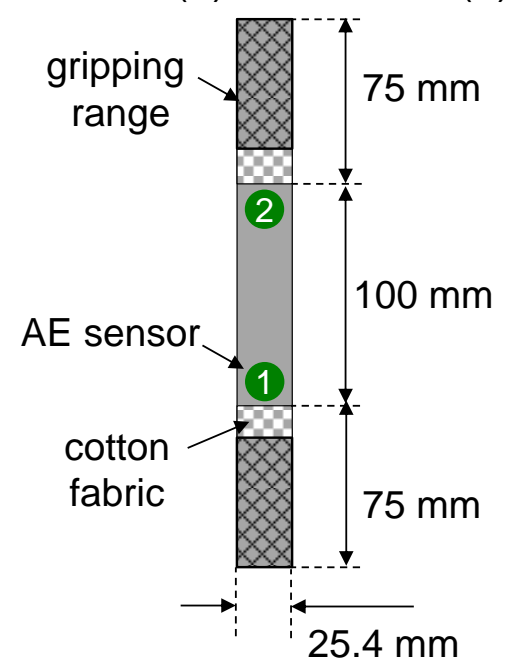

(b)

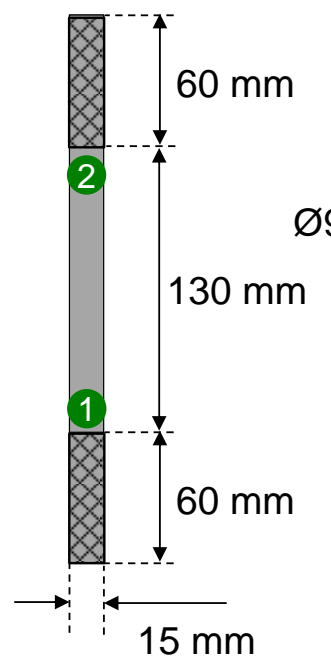

(c)

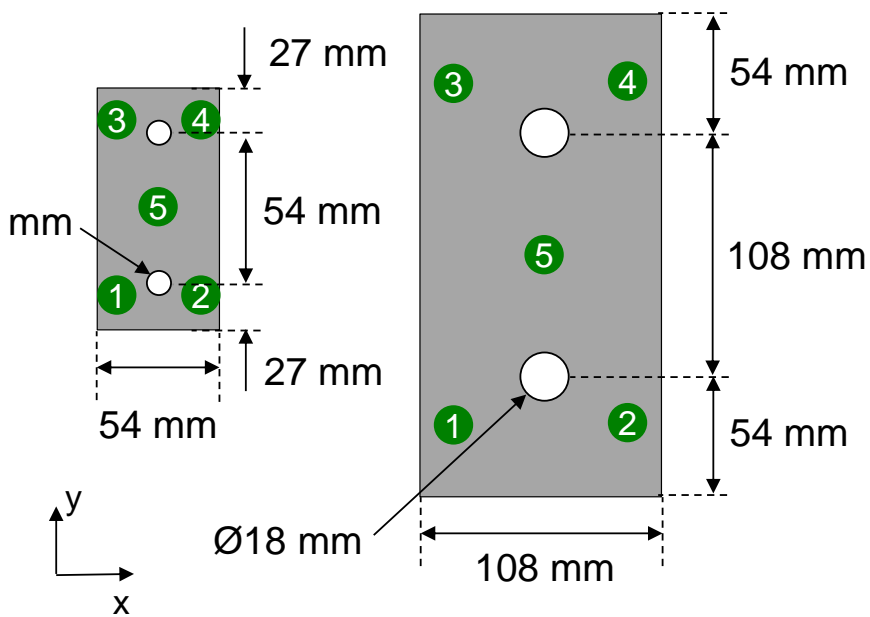

Figure 6: Test sample geometries investigated including acoustic emission sensor positions for tape tensile tests (a), tensile tests (b) and bearing strength test (c).

\subsection{Tensile tests on tape material}

For processing with automated tape laying, the T700 carbon fibers are embedded in the thermoplastic PPS matrix in form of tape material supplied with 1" $(25.4 \mathrm{~mm})$ width. The nominal fiber volume fraction of the tapes was evaluated as 0.57 . As first step of this study, these tapes are tested in the as-received state. For preparation of tensile test specimens, we use resin impregnated cotton fabrics as additional reinforcement in the clamping region (see figure 6-a). The room temperature curing adhesive system Stycast 2850 FT was used to bond specimens and reinforcement tabs. This yields an increase of nominal thickness from $0.16 \mathrm{~mm}$ in the test region to $0.95 \mathrm{~mm}$ in the reinforcement range. We choose a total length of $250 \mathrm{~mm}$ with $100 \mathrm{~mm}$ length reduced section and kept the $25.4 \mathrm{~mm}\left(1^{\prime \prime}\right)$ width of the tape for the test samples. Since unidirectional tapes were used, the fiber axis direction is parallel to the length direction of the specimen. The specimens were tested in displacement controlled mode with $2 \mathrm{~mm} / \mathrm{min}$ test speed using a universal testing machine with $50 \mathrm{kN}$ load cell and self-jamming wedge grips using complete loading and unloading cycles. Load increments were chosen as $200 \mathrm{MPa}$ load increments with intermediate unloading to $50 \mathrm{MPa}$ tensile stress at $10 \mathrm{~mm} / \mathrm{min}$ test speed.

\subsection{Tensile tests}

The aforementioned tape material was used to fabricate test laminates with six layers in unidirectional layup using in-situ laser consolidation. All specimens were cut to nominal specimen dimensions of $250 \mathrm{~mm} \times 15 \mathrm{~mm} \times 1 \mathrm{~mm}$ (length $\times$ width $\times$ thickness) with the fiber axis direction parallel to the length direction of the specimen (see figure 6-b). The room temperature curing adhesive system Stycast 2850 FT was used to bond specimens and reinforcement tabs with ( $\pm 45^{\circ}$ layup) to the heat pressed specimens. The specimens were tested in accordance with DIN EN 2561 in displacement controlled mode with $2 \mathrm{~mm} / \mathrm{min}$ test speed using a universal testing machine with $250 \mathrm{kN}$ load cell and hydraulic grips 
using complete loading and unloading cycles. Load increments were chosen as $200 \mathrm{MPa}$ with intermediate unloading to $50 \mathrm{MPa}$ tensile stress at $10 \mathrm{~mm} / \mathrm{min}$ test speed.

\subsection{Bearing strength}

For this part of the investigation, we carried out bearing strength tests on specimens of $108.0 \mathrm{~mm} \times$ $54.0 \mathrm{~mm} \times 5.7 \mathrm{~mm}$ (length $\times$ width $\times$ thickness) with $9.0 \mathrm{~mm}$ diameter bolts and a scaled version with $216.0 \mathrm{~mm} \times 108.0 \mathrm{~mm} \times 11.4 \mathrm{~mm}$ (length $\times$ width $\times$ thickness) with $18.0 \mathrm{~mm}$ diameter bolts (both geometries see figure 6-c). The load was introduced using clamping strips in the spirit of DIN EN 6037 (type 2 configuration). However, both bolts are same diameter, so no preference was given for failure on either side of the laminate. As for the tensile test specimens, these specimens were also built using automated tape laying with in-situ laser consolidation. The stacking sequence is using the directed configuration $(0,+45,-45,0,90)_{4 \text { sym }}$ for the regular size and $(0,+45,-45,0,90)_{8 s y m}$ for the scaled size. Testing was carried out in displacement controlled mode at $2 \mathrm{~mm} / \mathrm{min}$ test speed using a universal testing machine with $250 \mathrm{kN}$ load cell. The load schedule is a step-wise increase by $40 \mathrm{MPa}$ until failure with intermediate unloading to $20 \mathrm{MPa}$ tensile stress as lower limit.

\subsection{Acoustic emission settings}

In all experimental settings, acoustic emission sensors were mounted on the specimen using suitable clamp systems to ensure reproducible contact pressure between sensor and specimen. As acoustic couplant, viscous Korasilone silicone grease was used. All signals were amplified by $20 \mathrm{~dB}$ using a 2/4/6 preamplifier and are recorded with $10 \mathrm{MSP} / \mathrm{s}$ sampling rate and $35 \mathrm{~dB}_{\mathrm{AE}}$ threshold using the software AEwin. For all configurations, a bandpass filter from $20 \mathrm{kHz}$ to $1 \mathrm{MHz}$ was used. In all cases, the trigger settings were chosen as $10 \mu$ s for Peak-Definition-Time and $80 \mu$ s for Hit-Definition-Time. Based on the difference in the sound velocity and test geometry we empirically adjusted the Hit-Lockout-Time values for each test case to avoid triggering the same event multiple times. The values used are $300 \mu s$ for the tape tensile test, $1500 \mu$ s for the tensile test and $10000 \mu \mathrm{s}$ for the bearing strength test.

For the tensile test cases two acoustic emission sensors in linear arrangement were used (see figure 6-a and 6-b). To detect only AE signals with source positions located in the tapered area, an Event-DefinitionTime filter was used. The settings for this filter were adjusted for each specimen individually to avoid detection of sources outside the tapered range. For further processing, only signals localized in the tapered region by a classical $\Delta$ t-based algorithm were taken into account.

For the bearing strength testing, five acoustic emission sensors were used with the geometric arrangement seen in figure 6-c. In this test configuration, the presence of a bolt in the laminate and the anisotropy of the fiber-reinforced material cause strong disturbance of the acoustic wave field. Therefore, we apply a neural networks based source localization approach as described in [22] following the specific implications outlined in chapter 4.6 in [4] to obtain an average source localization accuracy 
of $3.3 \mathrm{~mm}$. As for the tensile test cases, only localized signals were taken into account for further processing.

\begin{tabular}{|l|c|l|c|}
\hline Name & $\begin{array}{l}\text { Samples } \\
\text { tested }\end{array}$ & $\begin{array}{l}\text { Volume } \\
\text { (length } \times \text { width } \times \text { thickness) }\end{array}$ & $\begin{array}{l}\text { Accumulated acoustic } \\
\text { emission energy }\end{array}$ \\
\hline Tensile test (tapes) & 6 & $(100 \times 25.4 \times 0.16) \mathrm{mm}^{3}=406.4 \mathrm{~mm}^{3}$ & $(1.8 \pm 1.7) \times 10^{-7} \mathrm{~J}$ \\
\hline Tensile test & 6 & $(130 \times 15 \times 1) \mathrm{mm}^{3}=1950 \mathrm{~mm}^{3}$ & $(1.5 \pm 1.5) \times 10^{-5} \mathrm{~J}$ \\
\hline $\begin{array}{l}\text { Bearing strength (regular } \\
\text { size) }\end{array}$ & 3 & $(108 \times 54 \times 5.7) \mathrm{mm}^{3}=33,242 \mathrm{~mm}^{3}$ & $(2.5 \pm 1.8) \times 10^{-3} \mathrm{~J}$ \\
\hline $\begin{array}{l}\text { Bearing strength (scaled } \\
\text { size) }\end{array}$ & 3 & $\begin{array}{l}(216 \times 108 \times 11.4) \mathrm{mm}^{3}= \\
265,939 \mathrm{~mm}^{3}\end{array}$ & $(7.3 \pm 1.5) \times 10^{-3} \mathrm{~J}$ \\
\hline
\end{tabular}

Table 1: Summary of test condition volumes and acoustic emission energies.

\section{Results}

In order to demonstrate our failure prediction concept, we first present exemplarily the load schedules and introduce the specific acoustic emission parameters used in this study. Next, we present a discussion of the predicted failure loads and the measured failure loads.

\subsection{Evaluation of acoustic emission parameters}

Within this study, we used a load scheme with load/unload cycles to failure. Maximum load capacity was estimated based on quasi-static measurements in each of the three test conditions. Load increments were then defined accordingly to reach 10 to 20 cycles before ultimate failure (i.e. rupture for the tensile test cases and exceedance of the maximum strength for the load bearing cases). Exemplary load-time curves superimposed with the simultaneously acquired acoustic emission signals are shown in figure 7a for a tensile test sample and in figure 7-b for a load bearing test.

An example for the typical acoustic emission evaluation result of the tensile test case is shown in figure 8. The calculated acoustic emission ratios are plotted on the vertical axis, while the horizontal axis is chosen as load ratio value $L R$. The Felicity ratio data is shown in figure 8 -a, with the values of the $\langle F R\rangle$ evaluation connected by straight lines for better visibility. As expected from the definition of the Felicity ratio values, the $F R 5$ starts first in each cycle, followed by the $F R 10, F R 15$ and $F R 20$, which is seen in the graph as a systematic shift of the numerical values from low to high. For the first five cycles evaluated, the overall number of acoustic emission hits is so low, that all $F R$ values are evaluated identically, as single cascades of acoustic emission hits practically appeared at the same load level. In the later cycles it is clearly observed, that the $\langle F R\rangle$ value acts as arithmetic mean value of the other $F R$ values.

The evaluation of the Shelby ratio follows the same style of presentation and is shown in figure 8-b. The arithmetic average $\langle S R\rangle$ is connected by straight lines to improve visibility. As for the Felicity ratio, a falling trend of $S R$ values is observed as function of load ratios. However, the Shelby ratio can only be 
evaluated for those cycles, which exhibit acoustic emission signals during the unloading step. As this was not the case for all cycles, there are less data points than for the Felicity ratio evaluation of figure 8-a. In addition, we evaluate energetic ratios as explained in section 2.2.3. We choose the signal amplitude in $\left[\mathrm{dB}_{\mathrm{AE}}\right]$ the absolute signal energy in $[\mathrm{aJ}]$ and the average signal level in $\left[\mathrm{dB}_{\mathrm{AE}}\right]$ to evaluate the load level of exceedance relative to the previous cycle. As seen in figure 8-c, these do provide a certain trend as function of $L R$, but this is not necessarily linear. Based on the slight scatter in between the three different ratios of ERAMP, ERENE and ERASL it is also clear, that these values are not identical attempts to express the acoustic energy release per cycle, but show a certain common trend.

(a)

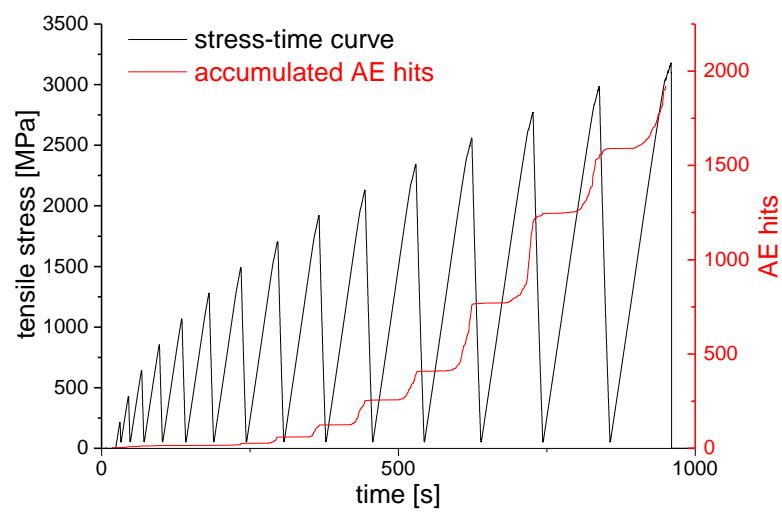

(b)

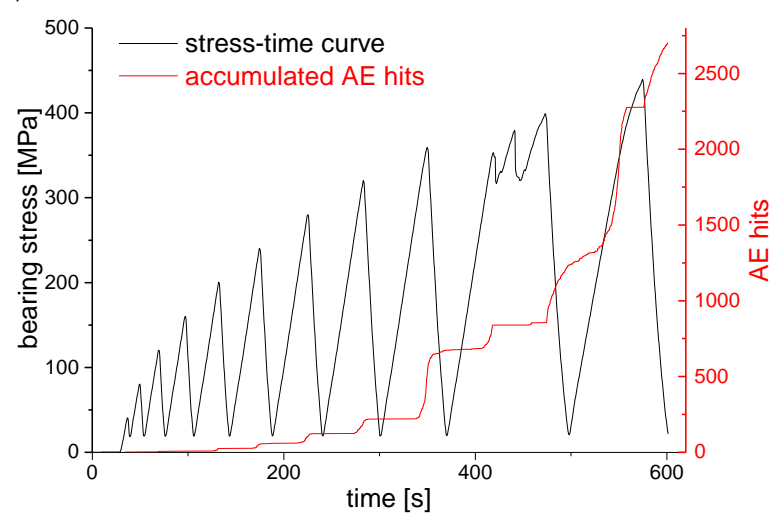

Figure 7: Exemplary load scheme for tensile test (a) and exemplary load scheme for load bearing test (b), both including accumulated number of acoustic emission hits.

(a)

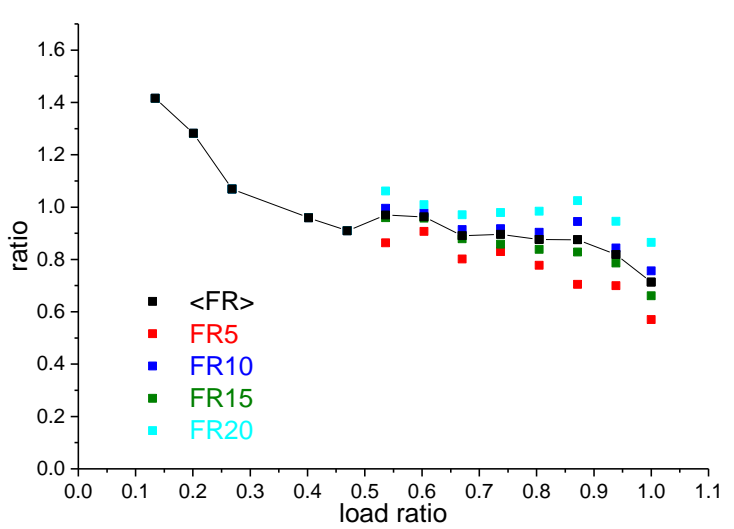

(c)

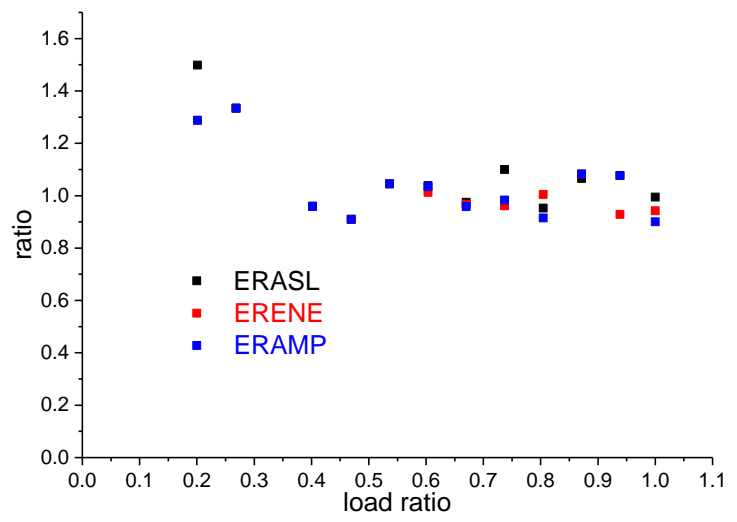

(b)

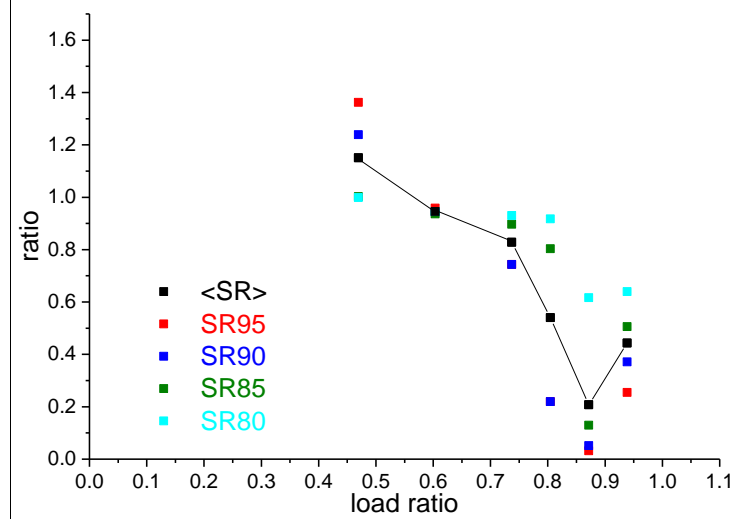

Figure 8: Felicity ratio evaluation for tensile test example of figure 8-a (a) and corresponding evaluation of Shelby ratio (b) and energetic ratios (c). 
In figure 9, an example for a load bearing test sample is shown using the same evaluations as in figure 8. Despite of some differences to the tensile test case, in all three figures there is a certain falling trend of acoustic emission ratios as function of load ratio. In figure 9-a the last cycle elucidates the susceptibility of the Felicity ratio, when it is based on a small number of first arriving signals. In the last cycle, the first acoustic emission signals appear already at 0.2 of the previous load, whereas the FR10, FR15 and FR20 indicate, that the primary onset is still above 0.5 . In such cases, the $\langle F R\rangle$ evaluation provides a good compromise between an early onset evaluation and susceptibility to the presence of potential outlier data. The Shelby ratio evaluation is shown in figure 9-b, which exhibits some more scatter than the Felicity ratios, but overall follows the same falling trend. For the data of figure 9-c, again a falling trend at the lowest three cycles is seen, which is similar to the tensile test case in figure 8-c. After settling in for some cycles, there is a fall-off towards the final cycles. This indicates once more the necessity for a non-linear regression analysis to extrapolate such trends for prediction of a valid load ratio.

(a)

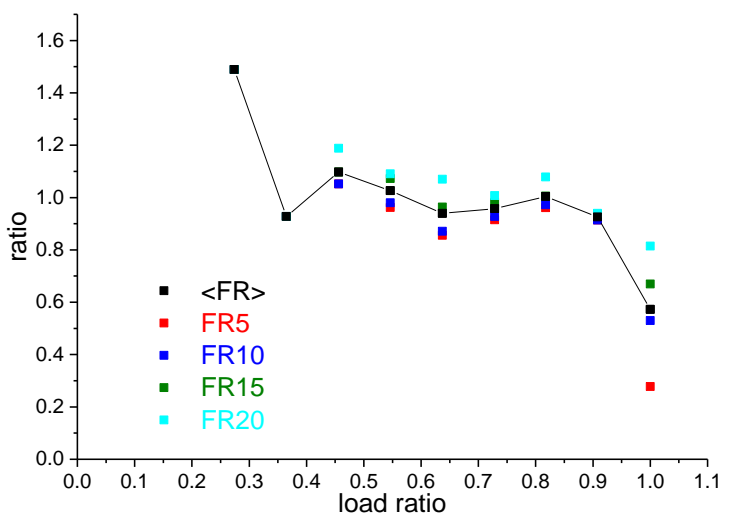

(c) (b)

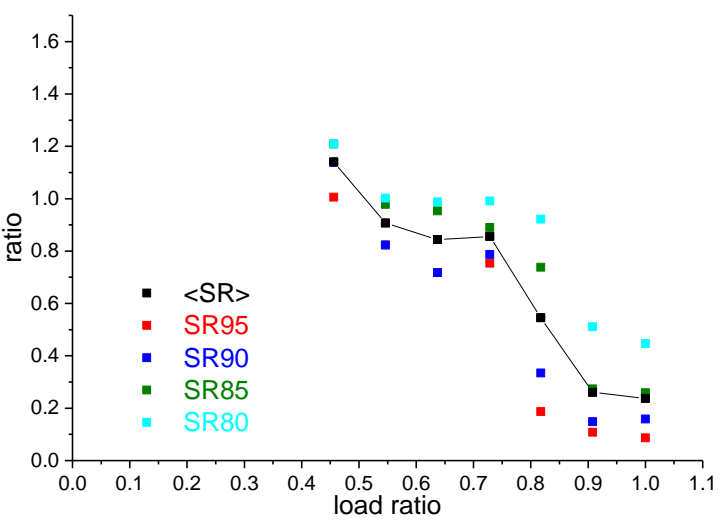

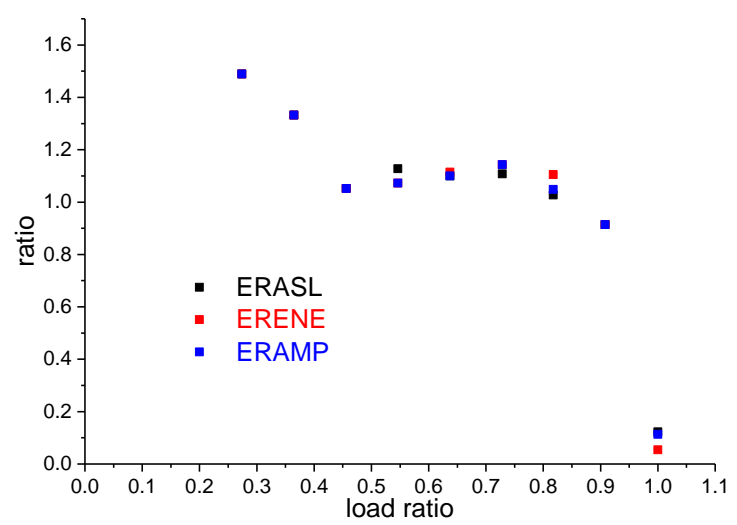

Figure 9: Felicity ratio evaluation for load bearing test example of figure 8-b (a) and corresponding evaluation of Shelby ratio (b) and energetic ratios (c).

As discussed in section 2.2 it appears necessary to define acoustic emission parameters for this kind of failure prediction as self-referencing values. As seen by comparison of the numerical values in figures 8 and 9 this was successfully reached by the ratio definitions of equations (2), (4) and (6). Despite of the volume change of more than two orders of magnitude between tensile samples and load bearing samples and the corresponding change in acoustic emission energy release of four orders of magnitude, 
the ratios span the same numerical ranges. This is crucial, if these ratios are to be used for a common data basis for predictive capabilities, such as predicting structure response based on coupon test data. Using absolute acoustic emission values would not suffice to this end, since absolute amplitude values, number of hits and alike are highly influenced by the type of load and the size of the test structure and the density of the sensor network.

In the following, these thirteen ratios were used to train the relationship between acoustic emission data and load ratios in order to perform the failure prediction outlined in the next section.

\subsection{Failure prediction}

In the following, we applied four different approaches to compare and discuss. For the failure prediction, we follow the extrapolation approach described in section 2.4. Two examples of this procedure are shown in figure 10 , one for tensile tests and one for the bearing strength test.

For all investigations, we define an upper limit of data points to use for the forward prediction. The data points excluded from the forward prediction are marked in gray in figures 10-a and 10-b. In addition, the prediction uncertainty is indicated by the red shaded cone. As there is zero predicted load at $L R=0$ as fixed boundary constraint, this reduces the uncertainty at values close to zero load. As seen in both figures, the scatter of data points is responsible for the prediction uncertainty. For the tensile test case we selected the result of sample 2 (cf. figure 12) to show a representative case for a reasonably high scatter in the prediction value. In this case, the deviation between predicted load and measured load is quite large with $7.0 \%$.

In comparison, the example for the bearing strength test is the result of sample 3, which exhibits less scatter. Here the deviation between predicted load and measured load is only $2.3 \%$. For the given two cases, the extrapolated linear relationship is marked as red line, with the predicted strength value shown as red square at the intersection between the predicted $L R=1$ and the linear extrapolation. As reference, the true failure strength is shown as green square and the dashed black line marks the real linear relationship between the applied stress and the $L R$ values. Both cases are prediction examples for the artificial neural network approach described in section 4.2.3.

(a)

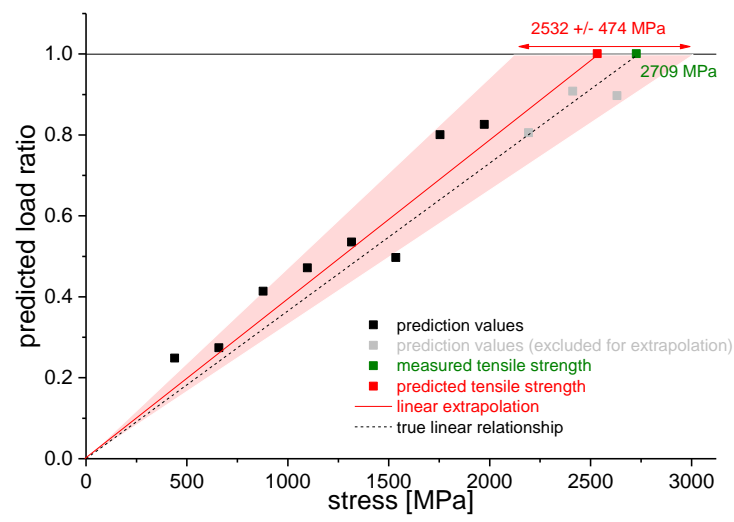

(b)

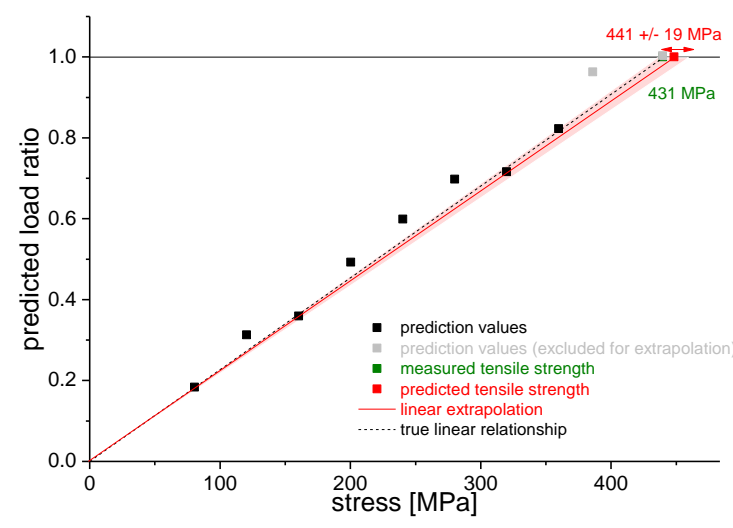


Figure 10: Extrapolation of predicted load ratios to yield failure strength for tensile test sample 2 (a) and load bearing test sample 3 (b).

For better comparability, we compiled all prediction results in figures 11,12 and 13, one for each test configuration. Besides the failure prediction results, these figures show the actual value of failure strength as solid gray horizontal line as reference.

\subsubsection{Same species, all samples ( $L R<0.85)$}

This concept represents a simple test case, since only identical geometries and test settings are considered. For this failure prediction, only training data of the same species is taken into account and all six samples are used as data basis. The purpose of this approach is the use of only one artificial neural network with 2 hidden layers and 5 neurons each, so prediction quality can be directly compared within one series of species. However, this is not understood as "true" prediction as the sample to be predicted is already included in the training data set, which is not a good practice. The resultant prediction values are shown as black squares in figures 11,12 and 13, with the extrapolation uncertainty added as error bars. For this investigation we choose an upper limit for the extrapolation basis of $L R<0.85$, meaning that the minimum extrapolation length is $15 \%$ of the samples ultimate tensile strength. The exact limit is specific for each sample, but ranges from $0.80<L R<0.85$. For this approach, given a higher number of artificial neurons and higher number of hidden layers, predicted LR values could be fitted exactly to their real $L R$ values. However, this would be considered an attempt of data overfitting, which is generally to avoid in using artificial neural networks. Thus, we used the same neural network structure as in section 4.2.2, to yield a representative result for this part of the study. Accordingly, the predicted values match the real failure strength values reasonably well. Within the margin of error, all sample failure strength values are predicted. However, the exact prediction value as well as the size of the error bars appears unique for every sample, as this mostly depends on the scatter of the available data points as seen in figure 10. This proves that the prediction concept is generally feasible.

\subsubsection{Same species, all but present sample (LR<0.85)}

In order to remove the main limitation of the previous approach, multiple artificial neural networks are now trained and evaluated. For every sample, the training data set is revised and all data points from the sample currently under investigation are removed. Subsequent to that, the 18 artificial neural networks are trained following the same rules as before using a network structure with 2 hidden layers and 5 neurons each. For each of the figures 11, 12 and 13, the training data basis is five samples of same species. The predicted failure strength is shown as red squares with corresponding error bars to represent the prediction uncertainty. We choose again an upper limit for the extrapolation basis of $L R<$ 0.85. For this approach, we find a valid prediction result for all specimens within the margin of error. This approach is considered as "true" prediction, as the artificial neural network training data does not 
include a-priori information of the specimen predicted. However, the training quality is highly unique for the different artificial neural networks, which is partially responsible for the large error bars. In addition, the smaller data basis (five instead of six samples) does not add to the stability of the procedure. However, this approach provides evidence, that the prediction of unknown sample properties is possible, given there is a sufficient number of identical type previously tested.

\subsubsection{All species, all but present sample ( $L R<0.85)$}

As next step, we mix the training data of all samples of all load cases and remove solely the data points of the sample to be predicted. Therefore, another 18 artificial neural networks with 2 hidden layers and 10 neurons each are trained and their results are shown in figures 11,12 and 13 as blue squares with prediction uncertainty as error bars and $L R<0.85$ as extrapolation limit. For each artificial neural network, the training data basis are 17 samples. Except for tape sample 2, the mixed training data set is capable of predicting the behavior in all three configurations quite well. For tape sample 2, the predicted value is slightly outside its margin of error, but the predicted value of $1449 \mathrm{MPa}$ is actually very close to the true value of $1422 \mathrm{MPa}$. The main differences of prediction quality originates from the specific artificial neural network training quality and the corresponding accuracy reached in the forward prediction. This proves that the structure of the artificial neural network is flexible enough to compensate for the differences in evolution of acoustic emission parameters as function of $L R$ not only for different samples, but also for different load conditions and test volumes, given a suitable number of sensors is used to detect the acoustic emission.

\subsubsection{All species, all but present sample (LR<0.64)}

Finally, we keep the same artificial neural networks as used in the previous section 4.2 .3 and modify the extrapolation length. As an upper limit of $L R=0.85$ may seem not adequate for quality control of composite structures, we reduce the upper limit to $L R<0.64$, which was the best compromise between the availability of data points for extrapolation and reasonably low load ratio. The prediction results are shown in figures 11,12 and 13 as green squares with their prediction uncertainty as error bars. The number of data points used for the extrapolation is shown next to the prediction value in green. For the tape tensile tests, the increase in forward prediction to $36 \%$ ultimate strength results in values which still fit with the reference values within the margin of error. For sample 5, no data points could be evaluated below $L R=0.64$, so no prediction is made for this sample. For the tensile test samples of figure 12, only five out of six samples were predicted within the margin of error. Especially samples 2 and 3 are far away from the real strength value, yet their prediction error is extraordinarily high. Consequently, this indicates, that the particular choice of $L R$ in this case might not be sufficient for a stable prediction. For the load bearing tests shown in figure 13, four out of six samples are predicted systematically lower than the reference value of the ultimate bearing strength, the other two samples 
exhibit high scatter. This behavior can readily be understood from figure 10-b. For the lower number of cycles the slope appears to be steeper, so their extrapolation is expected to result in lower strength values. Including more data points at higher $L R$ values, than changes the slope, so it becomes less steep. We added the measured initial bearing strength value (defined as first load drop in the loaddisplacement curve) as green lines to figure 13. According to the test standards, this value is interpreted as load value of initial bearing failure of the bolt, i.e. the first significant formation of damage in the composite at the contact surface between bolt and laminate. This may readily be understood as failure as well. Apparently, the prediction values at $L R<0.64$, coincide reasonably with the measured value for the first four samples. For the samples 5 and 6 , the prediction uncertainty is so high, that both values (ultimate strength and initial strength) fall into the prediction range. This is likely because the selected data points at around $L R=0.64$ either fall into the regime before initial bearing failure (samples 1-4) or include one more data point after initial bearing failure (samples 5-6). This is also the origin for the relatively high uncertainty of prediction. Overall, this final approach indicates, that reasonably low $L R$ Values can be used for forward prediction, given a suitable number of data points is still available and the failure mode retains unchanged.

In a real application with a maximum proof load of $0.64 L R$ one would rather modify the load schedule to yield 10-20 cycles in the load range between first onset of acoustic emission (e.g. $L R=0.2$ ) and $L R=0.64$ to obtain a sufficient number of data points for the linear extrapolation. Given similar accuracy of the predicted $L R$ values is reached as in the example of figure 10-b, this modified procedure should stabilize the prediction accuracy. However, the low acoustic emission activity at low $L R$ values needs to be taken into account for the selection of the number of cycles, as to few signals per load cycle may easily lead to erroneous predicted $L R$ values and could cause less stable prediction.

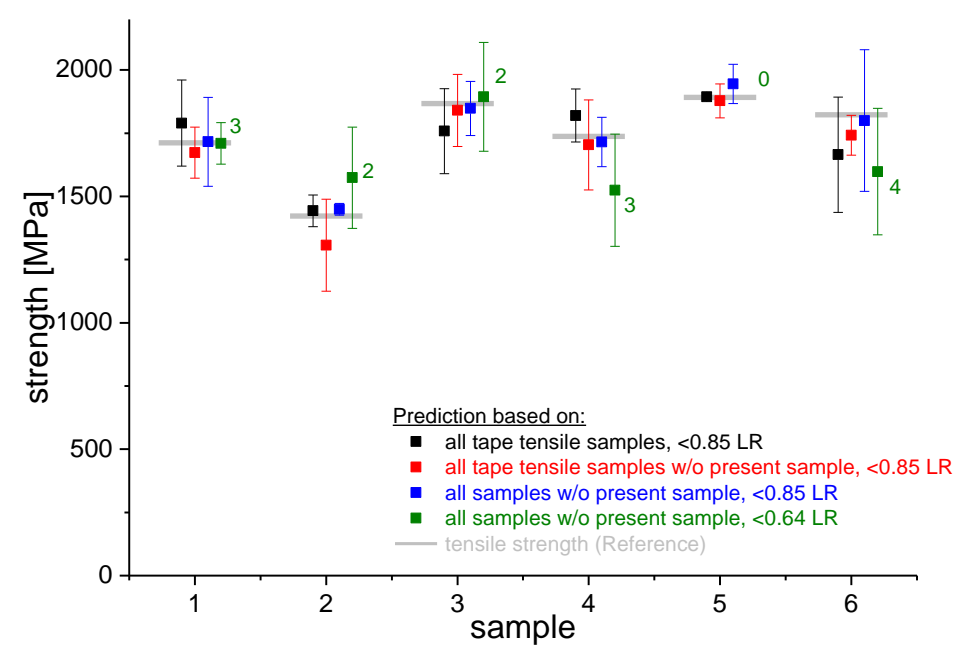

Figure 11: Forward prediction result of all tape tensile tests using different approaches to generate the training dataset for the artificial neural network. 


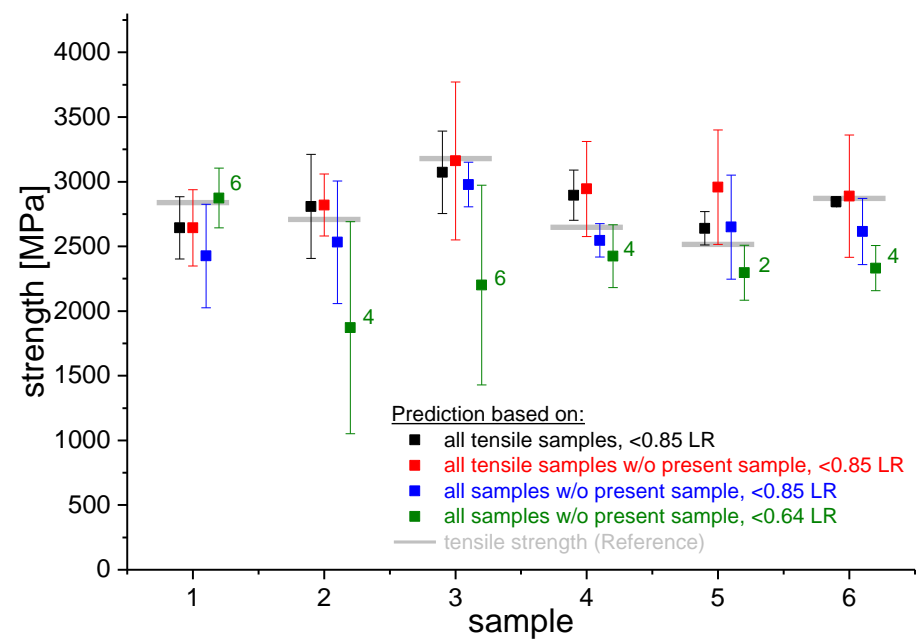

Figure 12: Forward prediction result of all tensile tests using different approaches to generate the training dataset for the artificial neural network.

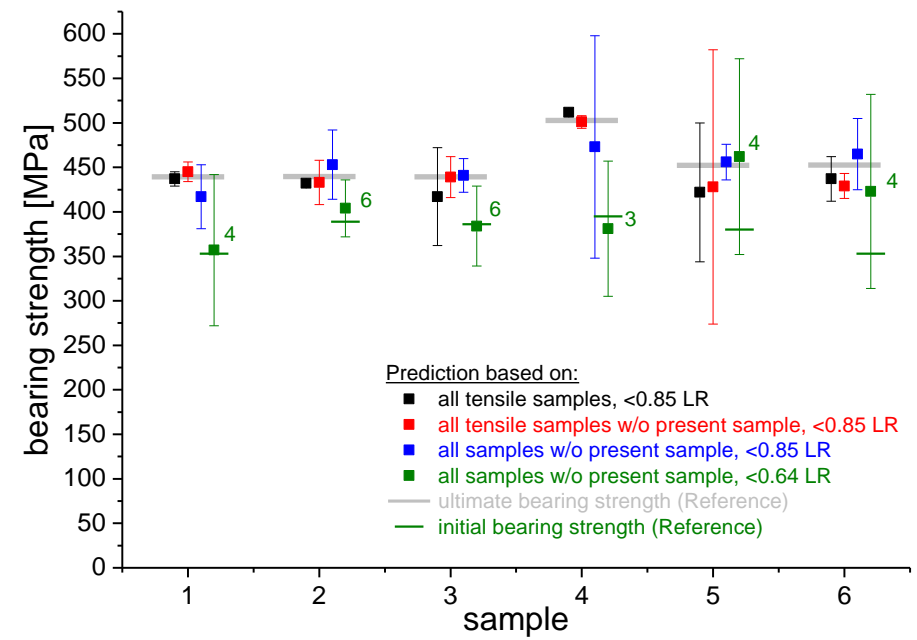

Figure 13: Forward prediction result of all bearing strength tests using different approaches to generate the training dataset for the artificial neural network.

\section{Conclusion}

Within this study, we outlined a concept to perform acoustic emission based failure prediction of fiberreinforced composites. The prediction is based on acoustic emission ratios evaluated in load-unload cycles. The ratios are defined independent of the absolute number of acoustic emission hits. With the presented case studies, it appears feasible to allow forward prediction for specimen strength values, given a suitable number of cycles is used. Clearly, the forward prediction routine benefits from a high number of load-unload cycles, but in a real test situation, a compromise between duration of the test campaign and the number of cycles is required. For the 10 to 20 cycles used in this study, the prediction values were found to coincide with the real strength value within the margin of error. This was regardless of the specific artificial neural network approach taken. Five test samples proved sufficient for the prediction of strength values. However, as the approach is based on a supervised training stage, the prediction accuracy would benefit from a larger number of test cases than used in this study. 
Using a combination of all samples, the mean prediction accuracy with reference to the ultimate strength of the samples is $0.2 \%$ for the tape tensile tests, $1.3 \%$ for the classical tensile tests and $0.7 \%$ for the load bearing tests at a forward prediction limit of $L R=0.85$. This is much lower than the typical coefficient of variation within the sample series, which evaluates as $9.8 \%$ for the tape tensile tests, $8.2 \%$ for the classical tensile tests and $5.4 \%$ for the load bearing tests. The prediction uncertainty evaluates as $7.1 \%$ for the tape tensile tests, $11.8 \%$ for the classical tensile tests and $10.1 \%$ for the load bearing tests when taking the ultimate strength as reference value.

A reduction of forward prediction limit to $L R=0.64$ results in slightly worse values of $2.6 \%$ for the tape tensile tests, $5.7 \%$ for the classical tensile tests and $0.2 \%$ for the load bearing tests when excluding the samples with uncertainties of more than $50 \%$ the nominal value and referencing to the initial bearing strength. The corresponding mean prediction uncertainty evaluates as $11.4 \%$ for the tape tensile test, $8.0 \%$ for the tensile test and $15.8 \%$ for the bearing strength test.

The prediction values as well as the prediction accuracy depends significantly on the quality of the input data, i.e. the acoustic emission ratios. Other definitions and approaches then presented in section 2.2, which lead to a more stable evaluation of the acoustic emission ratios are worthwhile to be considered for future approaches. Other factors, which could affect the acoustic emission release relative to the test data basis, should be carefully assessed as well. Among these, the research on different load concepts and the effect of temperature should be analyzed to understand its impact on the evaluation of the acoustic emission ratios. However, especially for equivalent load conditions, identical specimen geometries and otherwise equal measurement conditions (e.g. instrumentation, threshold, preamplifier gain, trigger settings, ...), the presented approach is expected to safely predict the failure strength of composite materials.

\section{Acknowledgments}

We would like to thank Marvin A. Hamstad for the discussions on the acoustic emission ratios and the scientific exchange on this research topic as well as the Free State of Bavaria for funding the project ComBo within the program "BayernFIT".

\section{Literature}

[1] A.S. Kaddour, M.J. Hinton, Maturity of 3D failure criteria for fibre-reinforced composites: Comparison between theories and experiments: Part B of WWFE-II, J. Compos. Mater. 47 (2013) 925-966. doi:10.1177/0021998313478710.

[2] P.D. Soden, a. S. Kaddour, M.J. Hinton, Recommendations for designers and researchers resulting from the world-wide failure exercise, Compos. Sci. Technol. 64 (2004) 589-604. doi:10.1016/S0266-3538(03)00228-8. 
[3] A.S. Kaddour, M.J. Hinton, P.D. Soden, A comparison of the predictive capabilities of current failure theories for composite laminates: Additional contributions, Compos. Sci. Technol. 64 (2004) 449-476. doi:10.1016/S0266-3538(03)00226-4.

[4] M.G.R. Sause, In Situ Monitoring of Fiber-Reinforced Composites, Springer International Publishing, Cham, 2016. doi:10.1007/978-3-319-30954-5.

[5] M. Sause, M. Hamstad, Acoustic Emission Analysis, in: P.W.R. Beaumont, C.H. Zweben (Eds.), Compr. Compos. Mater. II, Elsevier, Oxford, 2018: pp. 291-326. doi:10.1016/B978-0-12-8035818.10036-0.

[6] J. Kaiser, Untersuchungen über das Auftreten von Geräuschen beim Zugversuch, Dissertation, Technische Hochschule München, 1950.

[7] T.J. Fowler, Acoustic Emission Testing of Fiber Reinforced Plastics, in: Prepr. 3092, American Society of Civil Engineers, New York, 1977.

[8] T.J. Fowler, Acoustic Emission Testing of Fiber Reinforced Plastics, Proc. Pap. J. Tech. Counc. ASCE. 105(TC2) (1979) 281-289.

[9] K.S. Downs, M.A. Hamstad, Acoustic Emission from Depressurization to Detect/Evaluate Significance of Impact Damage to Graphite/Epoxy Pressure Vessels, J. Compos. Mater. 32 (1998) 258-307. doi:10.1177/002199839803200304.

[10] K.S. Downs, M.A. Hamstad, Correlation of Acoustic Emission Felicity Ratios and Hold-Based Rate Moments with Burst Strngths of Spehrical Graphite/Epoxy Pressure Vessels, J. Acoust. Emiss. 13 (1995) 45-56.

[11] T.J. Fowler, Revisions to the CARP Recommended Practice for Tanks and Vessels, in: Proc. Fifth Int. Symp. Acoust. Emiss. From Compos. Mater. - AECM-5, American Society for Nodestructive Testing, Columbia, Ohio, Sundsvall, Sweden, 1995: pp. 263-271.

[12] J.M. Waller, C.T. Nichols, D.J. Wentzel, R.L. Saulsberry, D.O. Thompson, D.E. Chimenti, Use of Modal Acoustic Emission to Monitor Damage Progression in Carbon Fiber/Epoxy Composites, in: AIP Conf. Proc., San Diego, California, (USA), 2011: pp. 919-926. doi:10.1063/1.3592036.

[13] E.K. Hill, S.T. Dion, J.O. Karl, N.S. Spivey, J.L.W. Ii, Neural Network Burst Pressure Prediction in Composite Overwrapped Pressure Vessels, Neural Networks. 25 (2007) 187-193.

[14] J.L. Walker, E.V.K. Hill, Backpropagation neural networks for predicting ultimate strengths of unidirectional graphite/epoxy tensile specimens, Adv. Perform. Mater. 3 (1996) 75-83. doi:10.1007/BF00136861.

[15] J.L. Walker, G.L. Workman, S.S. Russell, E.V.K. Hill, Neural network/acoustic emission burst pressure prediction for impact damaged composite pressure vessels, Mater. Eval. 55 (1997). http://www.osti.gov/scitech/biblio/562042 (accessed May 2, 2015).

[16] S. Rajendraboopathy, T. Sasikumar, K.M. Usha, E.S. Vasudev, Artificial neural network a tool for 
predicting failure strength of composite tensile coupons using acoustic emission technique, Int. J. Adv. Manuf. Technol. 44 (2009) 399-404. doi:10.1007/s00170-008-1874-x.

[17] H.M. Deuschle, A. Puck, Application of the Puck failure theory for fibre-reinforced composites under three-dimensional stress: Comparison with experimental results, J. Compos. Mater. 47 (2013) 827-846. doi:10.1177/0021998312462158.

[18] A. Puck, Failure analysis of FRP laminates by means of physically based phenomenological models, Compos. Sci. Technol. 58 (1998) 1045-1067. doi:10.1016/S0266-3538(96)00140-6.

[19] A.R.A. Abraham, K.L. Johnson, C.T. Nichols, R.L. Saulsberry, J.M. Waller, Use of Statistical Analysis of Acoustic Emission Data on Carbon-Epoxy COPV Materials-of-Construction for Enhanced Felicity Ratio Onset Determination - JSC-CN-26080, 2011. doi:10.13140/RG.2.1.1627.6005.

[20] T. Fowler, J. Blessing, F. Strauser, Intensity Analysis, in: Proc. Fourth Int. Symp. Acoust. Emiss. From Compos. Mater. - AECM-4, American Society for Nodestructive Testing, Columbia, Ohio, Seattle, 1992: pp. 237-249.

[21] C.M. Bishop, Neural Networks for Pattern Recognition, Clarendon Press, Oxford, 1995.

[22] S. Kalafat, M.G.R. Sause, Acoustic emission source localization by artificial neural networks, Struct. Heal. Monit. 14 (2015) 633-647. doi:10.1177/1475921715607408. 